\title{
Local Sensitivity Analysis of Kinetic Models for Cellulose Pyrolysis
}

\author{
(Accepted by Waste and Biomass Valorization)
}

Zhujun Dong ${ }^{\text {a }}$, Li Xie ${ }^{\text {a }}$, Yang Yang ${ }^{\mathrm{b}}$, Anthony V. Bridgwater ${ }^{\mathrm{b}}$, Junmeng Cai ${ }^{\mathrm{a}, *}$

${ }^{a}$ Biomass Energy Engineering Research Center, Key Laboratory of Urban Agriculture (South) Ministry of Agriculture, School of Agriculture and Biology, Shanghai Jiao Tong University, 800 Dongchuan Road, Shanghai 200240, People's Republic of China

${ }^{\mathrm{b}}$ Bioenergy Research Group, European Bioenergy Research Institute (EBRI), Aston University, Birmingham B4 7ET, United Kingdom

Corresponding author: Junmeng Cai. Tel.: +86-21-34206624; E-mail address: jmcai@sjtu.edu.cn.

\section{Graphical abstract:}

$$
\text { Kinetic Model } \frac{\mathrm{d} \alpha}{\mathrm{d} T}=\frac{1}{\beta} \exp \left(\ln A-\frac{E}{R T}\right)(1-\alpha)^{n}
$$

$$
\begin{gathered}
\text { Local sensitivity of } \alpha \\
\frac{\partial \alpha}{\partial p} \approx \frac{\Delta \alpha}{\Delta p}=\frac{\alpha(T, p+\Delta p)-\alpha(T, p-\Delta p)}{2 \Delta p}
\end{gathered}
$$

$\mid$\begin{tabular}{c|c|} 
Local sensitivity of $\mathrm{d} \alpha / \mathrm{d} T$ \\
$\frac{\partial \frac{\mathrm{d} \alpha}{\mathrm{d} T}}{\partial p} \approx \frac{\Delta \frac{\mathrm{d} \alpha}{\mathrm{d} T}}{\Delta p}=\frac{\frac{\mathrm{d} \alpha}{\mathrm{d} T}(T, p+\Delta p)-\frac{\mathrm{d} \alpha}{\mathrm{d} T}(T, p-\Delta p)}{2 \Delta p}$
\end{tabular}
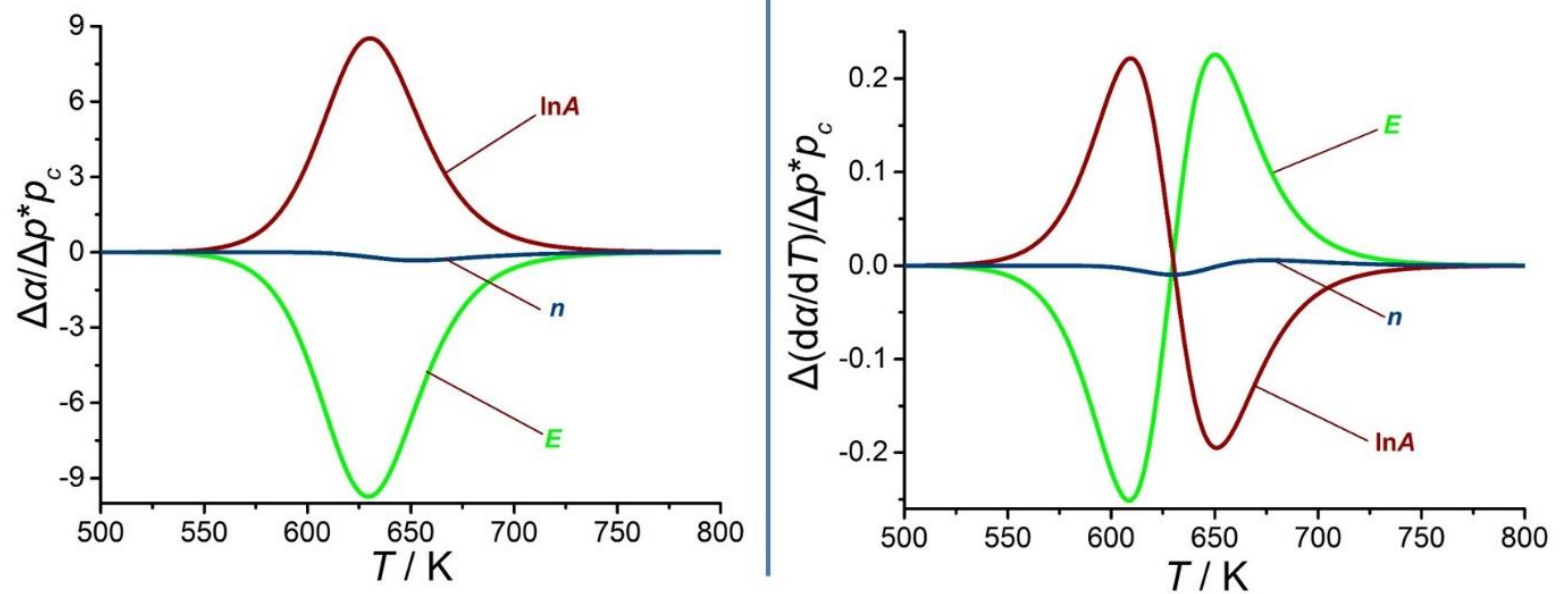


\begin{abstract}
:
The first and $n$th order kinetic models are usually used to describe cellulose pyrolysis. In this work, the local sensitivities of the conversion and derivative conversion with respect to the frequency factor, the logarithm of the frequency factor, the activation energy and the reaction order for the first and $n$th order kinetic models are calculated by using the finite difference method. The results show that the sensitivities of the first and $n$th order kinetic models with respect to the activation energy and the logarithm of the frequency factor are significant, while the frequency factor and the reaction order affect the $n$th order kinetic model slightly. Compared with the frequency factor, the natural logarithm of the frequency factor is a better choice in the parameter estimation of the first and $n$th order kinetic models.
\end{abstract}

Key words: Pyrolysis; Kinetic model; Local sensitivity analysis; Activation energy; Frequency factor

\title{
List of symbols:
}

ODE Ordinary differential equation

RRS Residual sum of squares

TG The thermo-gravimetric

DTG The derivative thermo-gravimetric

DSC Differential scanning calorimetry

Q-DTA Differential thermal analysis under quasi-isothermal, quasi-isobaric conditions

$\alpha \quad$ Degree of conversion

$n \quad$ Reaction order

A Frequency factor

$\ln A \quad$ Natural logarithm of frequency factor

E Activation energy

$R \quad$ Universal gas constant

$\beta \quad$ Heating rate 


$\begin{array}{ll}t & \text { Time } \\ T & \text { Absolute temperature. } \\ T_{0} & \text { Starting temperature } \\ p & \text { Kinetic parameter in the model } \\ n_{d} & \text { Number of data points }\end{array}$

\section{Subscripts:}

$\begin{array}{ll}\text { exp } & \text { Experimental data } \\ \text { cal } & \text { Calculated data } \\ i & \text { The } i \text { th data point } \\ c & \text { Corresponding kinetic parameter value }\end{array}$

\section{Introduction}

With the rapid growth of energy demand and increasing environmental concern, biomass has been widely used as an alternative source to fossil fuels due to its renewability, abundant reserves, and $\mathrm{CO}_{2}$ neutrality [1]. Pyrolysis is a thermochemical conversion process that decomposed organic matters in the absence of oxygen resulting in the production of pyrolysis liquids, char and permanent gases [2]. Cellulose is a major component of the lignocellulosic biomass. Understanding the kinetics of cellulose pyrolysis is important for effectively analyzing the conversion of biomass using pyrolysis-based technologies [3]. In the literature, cellulose pyrolysis was considered as a complicated process involving scission mechanism [4], intermediate species and phase change phenomena [5].

The thermo-gravimetric (TG), derivative thermo-gravimetric (DTG), differential scanning calorimetry (DSC) and differential thermal analysis under quasi-isothermal, quasiisobaric conditions (Q-DTA) could be used to perform the decomposition kinetic analysis of solid fuels [6-8]. The main aim of chemical kinetic study is to deduce the reaction mechanism governing the thermochemical reaction process, that is, to postulate a proper kinetic model and to determine the kinetic parameters correlated to the kinetic model [9]. The practical kinetic model with the estimated parameter values can be used for the prediction of the kinetic 
behaviors under different processing conditions. In general, there are several steps from the experimental phenomenon to the practical model including analysis of chemical mechanisms, sensitivity analysis and parameter estimation [10], as shown in Figure 1.

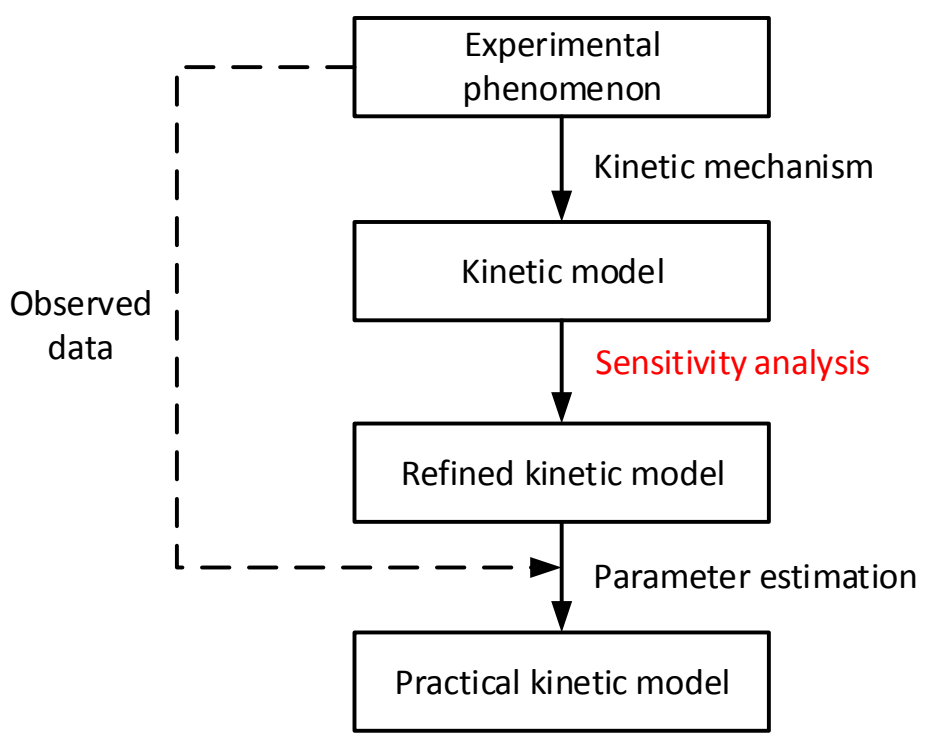

Figure 1. From experimental phenomenon to practical model

Sensitivity analysis is a technique used to study how the variation of kinetic parameters impacts the output of the kinetic model qualitatively and quantitatively [11]. It is a very useful tool to study the relationships between kinetic information flowing in and out of the reaction system, which can be used to enhance the model's confidence [12]. According to the literature [13], the residual sum of squares surface for some experimental data based on kinetic models shows a wide valley, which makes it difficult to find the optimal parameter values.

Many kinetic models have been proposed to describe the pyrolysis kinetics of cellulose [14], of which the first and $n$ th-order models were the most commonly used [15, 16]. Lin et al. [16] pointed out the first order kinetic model was able to adequately fit the kinetic experimental data of cellulose pyrolysis. Bradbury [17] considered that the initiation step of cellulose pyrolysis formed active cellulose followed by decomposing of active cellulose by two competitive first-order reactions. As shown in Figure 1, the sensitivity analysis of a chemical kinetic model is essential for the parameter estimation and the determination of the practical model. Sensitivity analysis can be divided into two categories: global and local sensitivity analysis. Global sensitivity analysis refers to the effect of simultaneous, possibly orders-ofmagnitude parameter changes, while local sensitivity analysis refers to small changes of parameters [18]. 
There were several studies related to the sensitivity analysis of kinetic models for biomass pyrolysis. Santos et al. [19] analyzed the influences of various kinetic parameters of the independent parallel reaction model for the pyrolysis of sugarcane bagasse by means of global sensitivity studies. The results showed that the activation energies of conversions had a stronger impact than other parameters such as the frequency factor and reaction order. Xavier et al. [20] performed the sensitivity analysis of the independent parallel reaction (IPR) model and obtained that the activation energies affected the conversion to a greater extent than the other parameters.

The above studies focused on the global sensitivity of kinetic models for biomass pyrolysis. The local sensitivities of the kinetic model outputs with respect to the kinetic parameters for the pyrolysis of biomass and its components are still missing. Therefore, the objective of this work is to measure local parametric sensitivity of the first and $n$th order kinetic models for cellulose pyrolysis. The results will be helpful to the pyrolysis of cellulose samples whose pyrolysis kinetic behaviors can be described by the first or $n$th order kinetic model. Besides, the corresponding analysis about cellulose pyrolysis is the first and basic step of investigating more complex reactions, such as the pyrolysis of lignocellulosic biomass or other solid fuels. Likewise, the approach about the relative sensitivity analysis presented in this work would provide a way to analyze other complex kinetic models, such as distributed activation energy model [21]. The corresponding sensitivity analysis of biomass pyrolysis is our next work which will use the results and approach of this work.

\section{The first order kinetic model and its numerical calculation}

The first order kinetic model is usually used to describe the global kinetics of cellulose pyrolysis and the corresponding kinetic parameters are apparent parameters [22]. The kinetic equation and the corresponding initial condition can be expressed as follows:

$$
\begin{gathered}
\frac{\mathrm{d} \alpha}{\mathrm{d} t}=A e^{-E / R T}(1-\alpha) \\
t=0, \quad \alpha=0
\end{gathered}
$$

where $\alpha$ is the degree of conversion, $t$ is the time, $A$ is the frequency factor, $E$ is the activation energy, $R$ is the universal gas constant, and $T$ is the absolute temperature.

There are three heating program types: isothermal heating program, non-isothermal heating program with a constant heating rate and other non-isothermal heating programs [23]. 
The second heating program is the most common in the experimental kinetic study of thermal decomposition processes. Under non-isothermal conditions at a constant heating rate, the following equation can be obtained:

$$
T=T_{0}+\beta t
$$

where $\beta$ is the heating rate and $T_{0}$ is the starting temperature.

According to Equation (3), Equations (1) and (2) become Equations (4) and (5), respectively.

$$
\begin{gathered}
\frac{\mathrm{d} \alpha}{\mathrm{d} T}=\frac{A}{\beta} e^{-E / R T}(1-\alpha) \\
T=T_{0}, \quad \alpha=0
\end{gathered}
$$

Before conducting sensitivity analysis, the solution of the kinetic model should be obtained. The problem of expressing $\alpha$ and $\mathrm{d} \alpha / \mathrm{d} T$ as a function of $T$ based on Equations (4) and (5) is a typical initial value problem for an ordinary differential equation (ODE). The analytical exact solution of the initial value problem for Equation (4) cannot be easily obtained, thus numerical techniques are frequently used for the solution of the problem [24]. In this work, the classical Runge-Kutta fourth order algorithm is selected for computing the initial value problem for ODEs. The implementation of the algorithm is carried out in the MATLAB software system.

Previous work carried out by Lin et al. [16] used the first order kinetic model to describe the kinetic behaviors of cellulose pyrolysis with a frequency factor of $6.46 \times 10^{14} \mathrm{~s}^{-1}$ and activation energy of $198.91 \mathrm{~kJ} \mathrm{~mol}^{-1}$. Using the same parameters, the influences of $\beta, A$ and $E$ on the numerical results of the first order kinetic model were revealed, as illustrated in Figure 2 (a), (b) and (c), respectively. The increase of $A$ causes the shift of $\alpha-T$ and $\mathrm{d} \alpha / \mathrm{d} T-T$ curves toward left direction. The $\alpha-T$ and $\mathrm{d} \alpha / \mathrm{d} T-T$ curves are shifted up the temperature scale by increases in $E$ and $\beta$. 

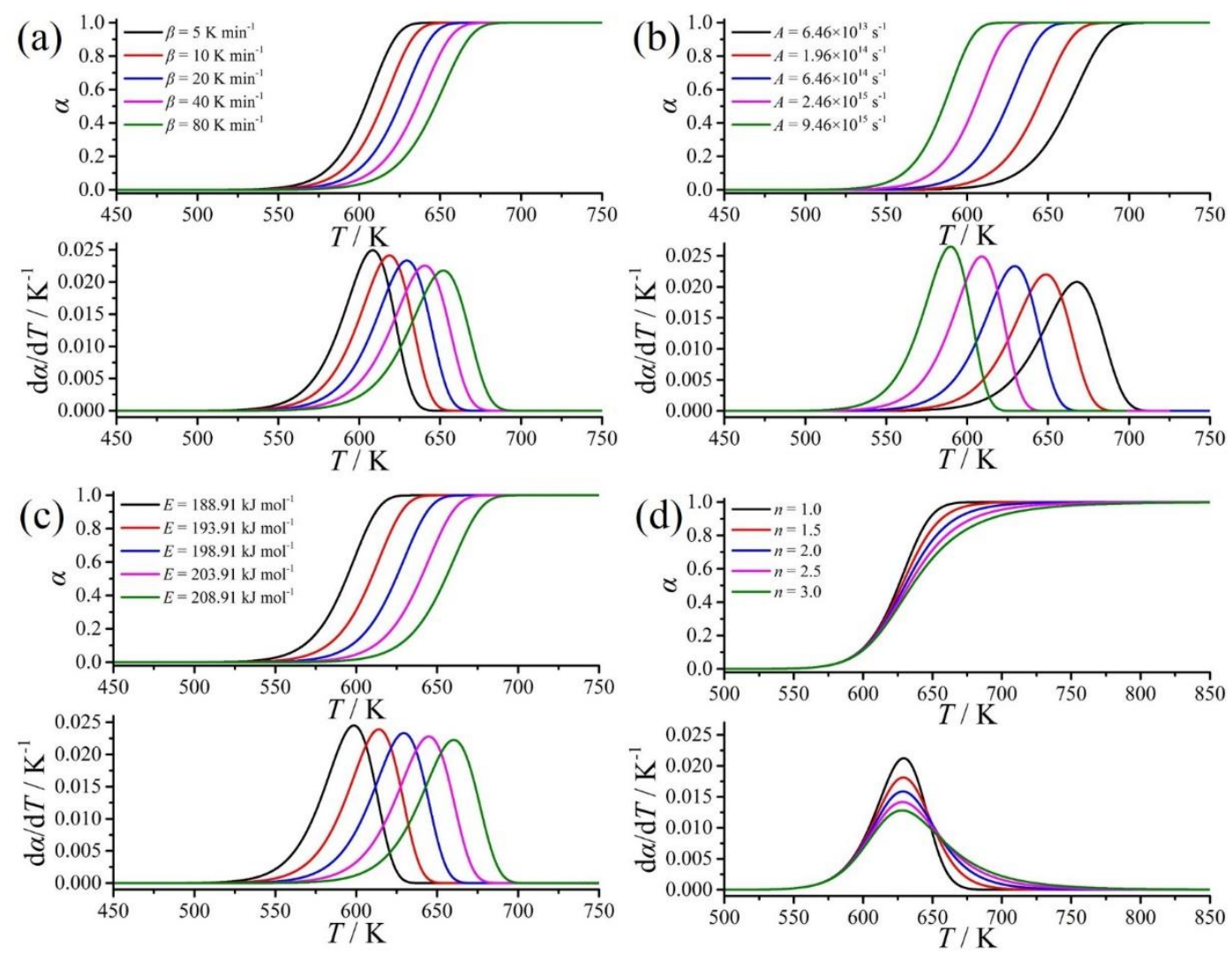

Figure 2. The effect of (a) $\beta\left(A=6.46 \times 10^{14} \mathrm{~s}^{-1}, E=198.91 \mathrm{~kJ} \mathrm{~mol}^{-1}\right.$ and $\left.n=1\right)$, (b) $A(\beta=20$ $\mathrm{K} \min ^{-1}, E=198.91 \mathrm{~kJ} \mathrm{~mol}^{-1}$ and $\left.n=1\right),(\mathrm{c}) E\left(\beta=20 \mathrm{~K} \mathrm{~min}^{-1}, A=6.46 \times 10^{14} \mathrm{~s}^{-1}\right.$ and $\left.n=1\right)$ and (d) $n\left(\beta=20 \mathrm{~K} \mathrm{~min}^{-1}, A=6.46 \times 10^{14} \mathrm{~s}^{-1}\right.$ and $\left.E=198.91 \mathrm{~kJ} \mathrm{~mol}^{-1}\right)$ on the numerical results of the first or $n$th order kinetic model

\section{Residual sum of squares surface for first order kinetic model}

Residual sum of squares (RSS) is a tool to measure the discrepancy between the data and an estimation model [25]. A small RSS indicates a tight fit of the model to the data [26]. It is usually used as an optimality criterion in parameter estimation.

In this work, the equations of RSS for $\alpha$ and $\mathrm{d} \alpha / \mathrm{d} T$ can be defined as

$$
\begin{gathered}
\mathrm{RSS}_{\alpha}=\sum_{i=1}^{n_{d}}\left(\alpha_{\mathrm{exp}, i}-\alpha_{\mathrm{cal}, i}\right)^{2} \\
\mathrm{RSS}_{\mathrm{d} \alpha / \mathrm{d} T}=\sum_{i=1}^{n_{d}}\left[\left(\frac{\mathrm{d} \alpha}{\mathrm{d} T}\right)_{\mathrm{exp}, i}-\left(\frac{\mathrm{d} \alpha}{\mathrm{d} T}\right)_{\mathrm{cal}, i}\right]^{2}
\end{gathered}
$$

where the subscripts exp and cal represent the experimental data and the data calculated from the kinetic model (4) respectively, $i$ represents the $i$ th data point, and $n_{d}$ represents the number 
of data points.

In order to investigate the effect of variation of $A$ and $E$ values on the RSS values, the theoretical $\alpha-T$ and $\mathrm{d} \alpha / \mathrm{d} T-T$ data with $A=6.46 \times 10^{14} \mathrm{~s}^{-1}$ and $E=198.91 \mathrm{~kJ} \mathrm{~mol}^{-1}$ (which were used to describe cellulose pyrolysis as quoted in Section 2) is used. The corresponding numerical calculations are performed in the MATLAB software system.

Figure 3 shows the RSS surfaces in the parameter space $\left(1.46 \times 10^{14} \mathrm{~s}^{-1} \leq A \leq 1.546 \times 10^{15}\right.$ $\mathrm{s}^{-1}$ and $188.91 \mathrm{~kJ} \mathrm{~mol}^{-1} \leq E \leq 208.91 \mathrm{~kJ} \mathrm{~mol}^{-1}$ ). From Figure 3, it can be found both the RSS surfaces for $\alpha$ and for $\mathrm{d} \alpha / \mathrm{d} T$ have a long valley, where the optimal parameter point is located. It is difficult to find the optimal parameter point in such a wide purple area. Therefore, we want to seek the key parameters whose input change would have more significant effects on the model output by sensitivity analysis.

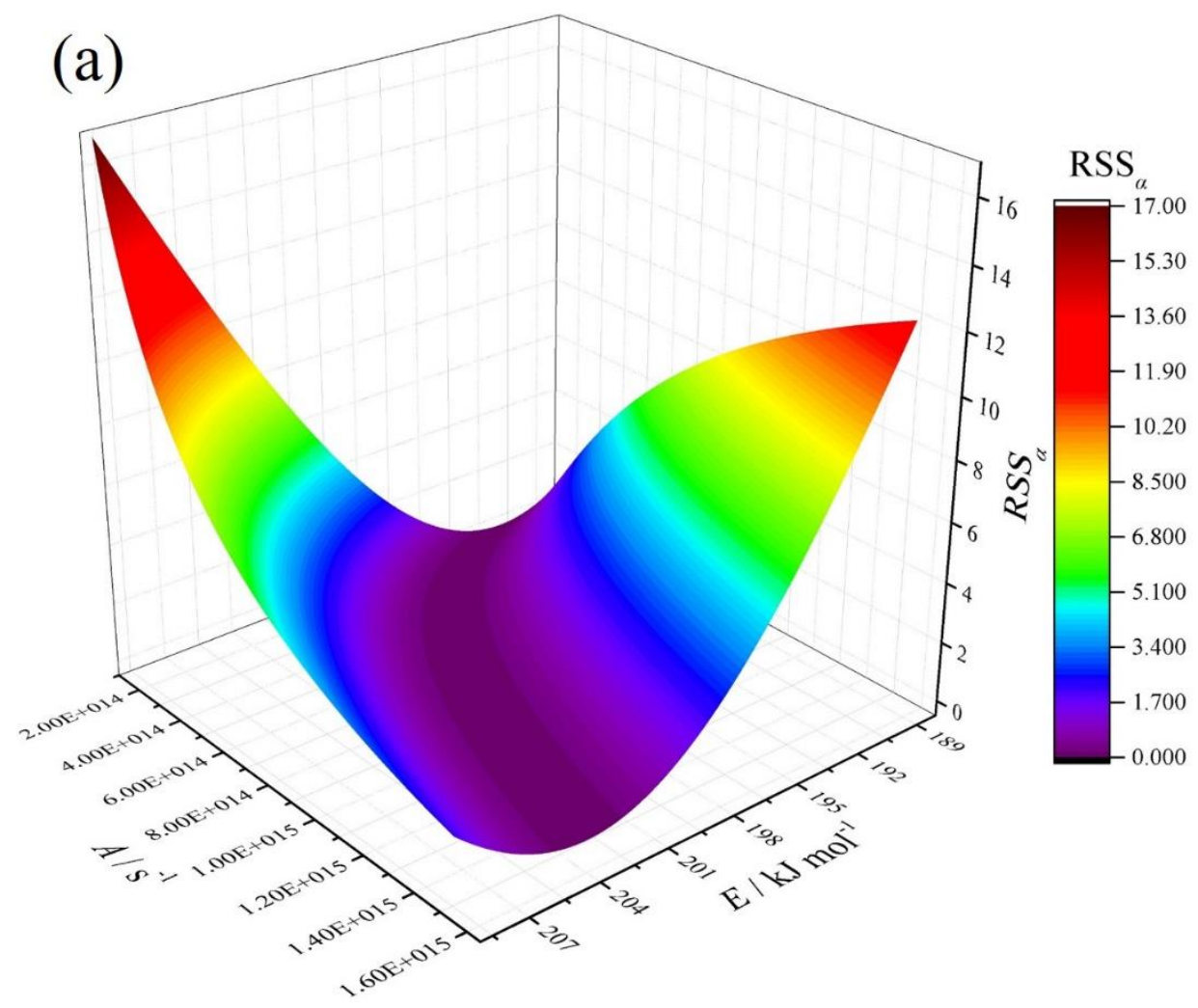




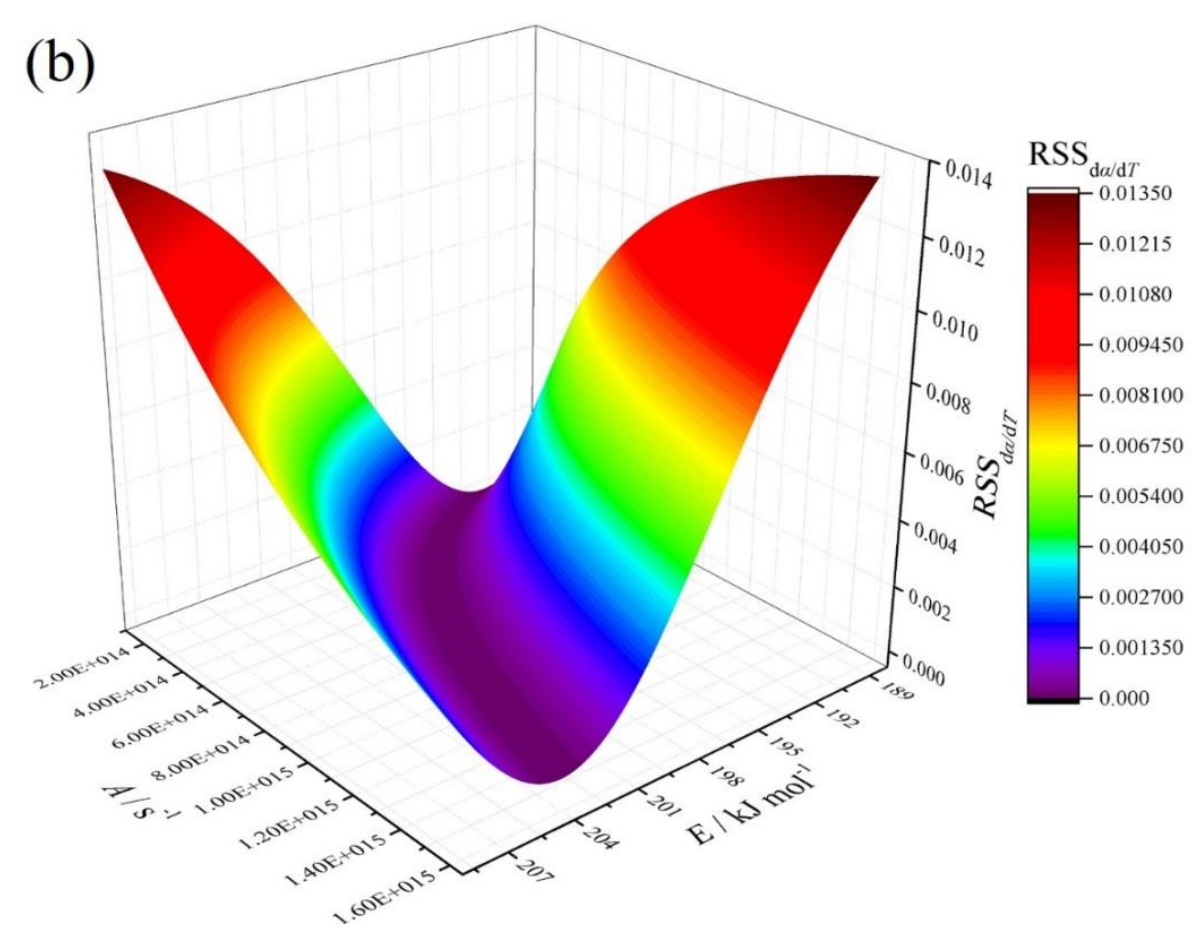

Figure 3. The RSS surfaces for (a) $\alpha$ and (b) $\mathrm{d} \alpha / \mathrm{d} T$ in the parameter space $\left(1.46 \times 10^{14} \mathrm{~s}^{-1} \leq A\right.$ $\leq 1.546 \times 10^{15} \mathrm{~s}^{-1}$ and $188.91 \mathrm{~kJ} \mathrm{~mol}^{-1} \leq E \leq 208.91 \mathrm{~kJ} \mathrm{~mol}^{-1}$ )

\section{Local sensitivity analysis of first order kinetic model}

There are many methods to perform sensitivity analysis, of which the finite difference method is used in this work for its simplicity [27]. Applying this method, the parameter $p$ is changed by $\Delta p$, while all other parameters remain unchanged. The sensitivities of $\alpha$ and $\mathrm{d} \alpha / \mathrm{d} T$ with respect to $A$ and $E$ for the first order kinetic model can be calculated by the following equations:

$$
\begin{gathered}
\frac{\partial \alpha}{\partial A} \approx \frac{\Delta \alpha}{\Delta A}=\frac{\alpha(T, A+\Delta A)-\alpha(T, A-\Delta A)}{2 \Delta A} \\
\frac{\partial \frac{\mathrm{d} \alpha}{\mathrm{d} T}}{\partial A} \approx \frac{\Delta \frac{\mathrm{d} \alpha}{\mathrm{d} T}}{\Delta A}=\frac{\frac{\mathrm{d} \alpha}{\mathrm{d} T}(T, A+\Delta A)-\frac{\mathrm{d} \alpha}{\mathrm{d} T}(T, A-\Delta A)}{2 \Delta A} \\
\frac{\partial \alpha}{\partial E} \approx \frac{\Delta \alpha}{\Delta E}=\frac{\alpha(T, E+\Delta E)-\alpha(T, E-\Delta E)}{2 \Delta E} \\
\frac{\partial \frac{\mathrm{d} \alpha}{\mathrm{d} T}}{\partial E} \approx \frac{\Delta \frac{\mathrm{d} \alpha}{\mathrm{d} T}}{\Delta E}=\frac{\frac{\mathrm{d} \alpha}{\mathrm{d} T}(T, E+\Delta E)-\frac{\mathrm{d} \alpha}{\mathrm{d} T}(T, E-\Delta E)}{2 \Delta E}
\end{gathered}
$$

The order of magnitude value of $A$ is relatively large (in general, $10^{10} \sim 10^{30} \mathrm{~s}^{-1}$ ) compared 
to that of $E$. Therefore, the natural $\operatorname{logarithm}$ of $A(\ln A)$ is usually considered in the parameter estimation. The sensitivities of $\alpha$ and $\mathrm{d} \alpha / \mathrm{d} T$ with respect to $\ln A$ can be expressed as follows:

$$
\begin{gathered}
\frac{\partial \alpha}{\partial \ln A} \approx \frac{\Delta \alpha}{\Delta \ln A}=\frac{\alpha(T, \ln A+\Delta \ln A)-\alpha(T, \ln A-\Delta \ln A)}{2 \Delta \ln A} \\
\frac{\partial \frac{\mathrm{d} \alpha}{\mathrm{d} T}}{\partial \ln A} \approx \frac{\Delta \frac{\mathrm{d} \alpha}{\mathrm{d} T}}{\Delta \ln A}=\frac{\frac{\mathrm{d} \alpha}{\mathrm{d} T}(T, \ln A+\Delta \ln A)-\frac{\mathrm{d} \alpha}{\mathrm{d} T}(T, \ln A-\Delta \ln A)}{2 \Delta \ln A}
\end{gathered}
$$

The above sensitivity calculations can be performed by using some numerical techniques. In this work, the MATLAB software system is used for the implementation of the finite difference method.

In this work, the relative sensitivity coefficient is used as a tool to measure the relative influence of parameters to model output avoiding the disturbance of magnitude. So that the different parameter sensitivities can be put together to compared with each other [19]. The relative sensitivity coefficients could be obtained by multiplying the absolute sensitivity coefficients by the parameter values: $\frac{\Delta \alpha}{\Delta A} A_{c}, \frac{\Delta \frac{\mathrm{d} \alpha}{\mathrm{d} T}}{\Delta A} A_{c}, \frac{\Delta \alpha}{\Delta E} E_{c}, \frac{\Delta \frac{\mathrm{d} \alpha}{\mathrm{d} T}}{\Delta E} E_{c}, \frac{\Delta \alpha(T)}{\Delta \ln A} \ln A_{c}$ and $\frac{\Delta \frac{\mathrm{d} \alpha}{\mathrm{d} T}}{\Delta \ln A} \ln A_{c} \quad\left(A_{c}\right.$ and $E_{c}$ represent the corresponding kinetic parameters for cellulose pyrolysis [16]). The relative sensitivity coefficient near zero means that a parameter change has small effect on the model output. As the relative sensitivity increases, the parameter changes in the model output become more significant [28].

The sensitivity analysis results based on the finite difference method are dependent on the parameter change. Figure 4 gives an example of the sensitivity of $\mathrm{d} \alpha / \mathrm{d} T$ with respect to $E$ with different $\Delta E$ values for the first order kinetic model.

From Figure 4(a), it can be observed that the temperature evolution of the sensitivity of d $\alpha / \mathrm{d} T$ with respect to $E$ would converge into a curve with decreasing of the $\Delta E$ values. The large $\Delta E$ value would damage the assumption of local linearity described in Equations (10) and (11). The smaller the $\Delta E$ value is, the closer the $\frac{\Delta \alpha}{\Delta A}$ and $\frac{\Delta \frac{\mathrm{d} \alpha}{\mathrm{d} T}}{\Delta E}$ values are to $\frac{\partial \alpha}{\partial A}$ and $\frac{\partial \frac{\mathrm{d} \alpha}{\mathrm{d} T}}{\partial E}$, respectively. From Figure 4(b), it can be seen that when the $\Delta E$ values are too small, 
the obtained sensitivity curves are not smooth. The small $\Delta E$ values would lead to relatively large computational round-off errors.

Therefore, there exist optimal values of parameter changes. Based on the calculated results, the optimal values of $\Delta E, \Delta A$ and $\Delta \ln A$ at $A_{c}=6.46 \times 10^{14} \mathrm{~s}^{-1}$ and $E_{c}=198.91 \mathrm{~kJ} \mathrm{~mol}^{-1}$ for the first order kinetic model are obtained: $\Delta E=0.2 \mathrm{~kJ} \mathrm{~mol}^{-1}, \Delta A=10^{12} \mathrm{~s}^{-1}$, and $\Delta \ln A=0.02$. Figure 5 shows the sensitivity analysis results of the first order kinetic model with the optimal parameter changes. Table 1 lists the corresponding peak values of the temperature evolution of the relative sensitivities for the first order kinetic model. From Figure 5 and Table 1, it can be observed that $\alpha$ and $\mathrm{d} \alpha / \mathrm{d} T$ show a strong sensitivity to $\ln A$ and $E$, but a slight sensitivity to $A$. This indicates that $\ln A$, instead of $A$, is better to be considered to use in the parameter estimation for the first order kinetic model.

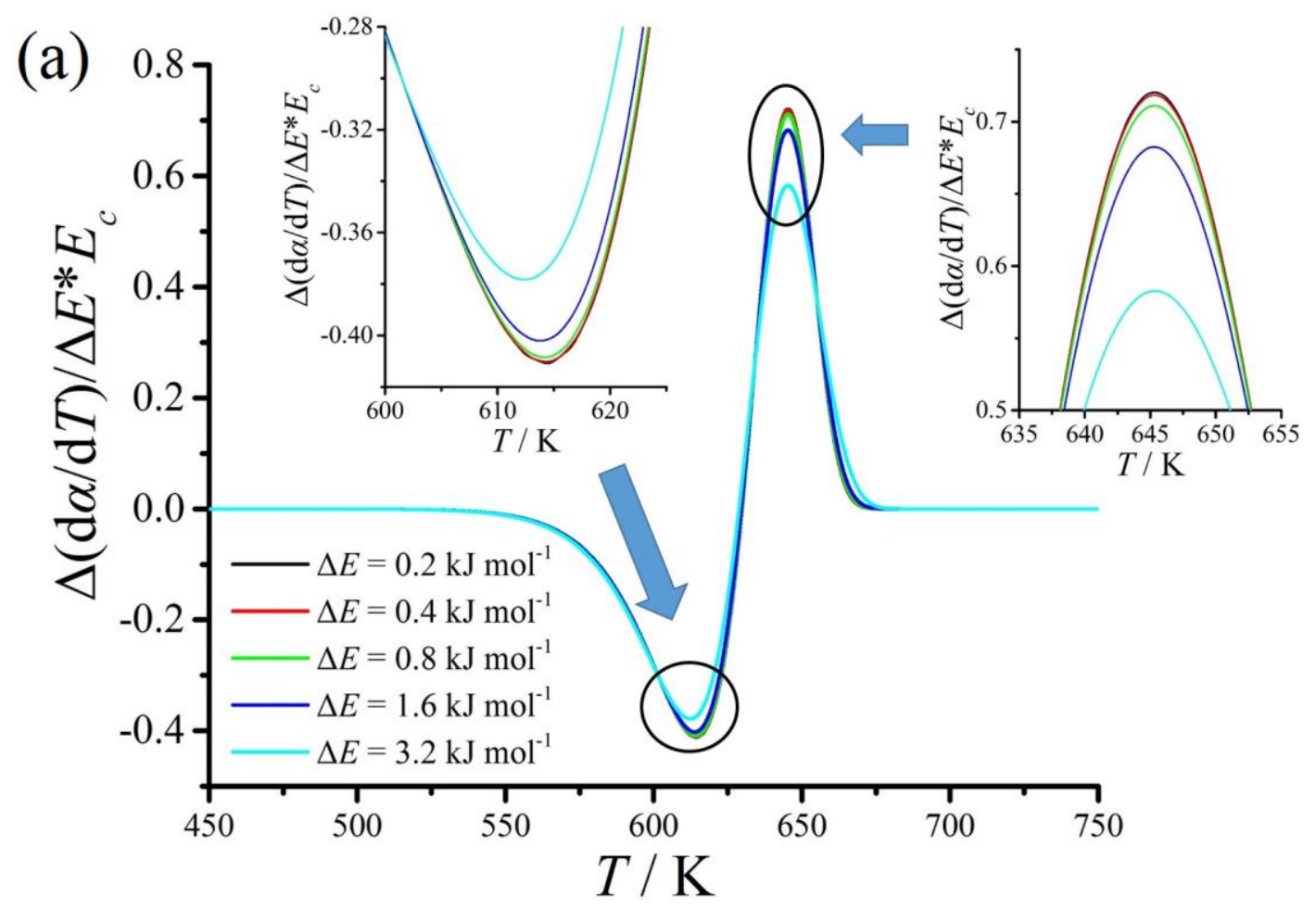




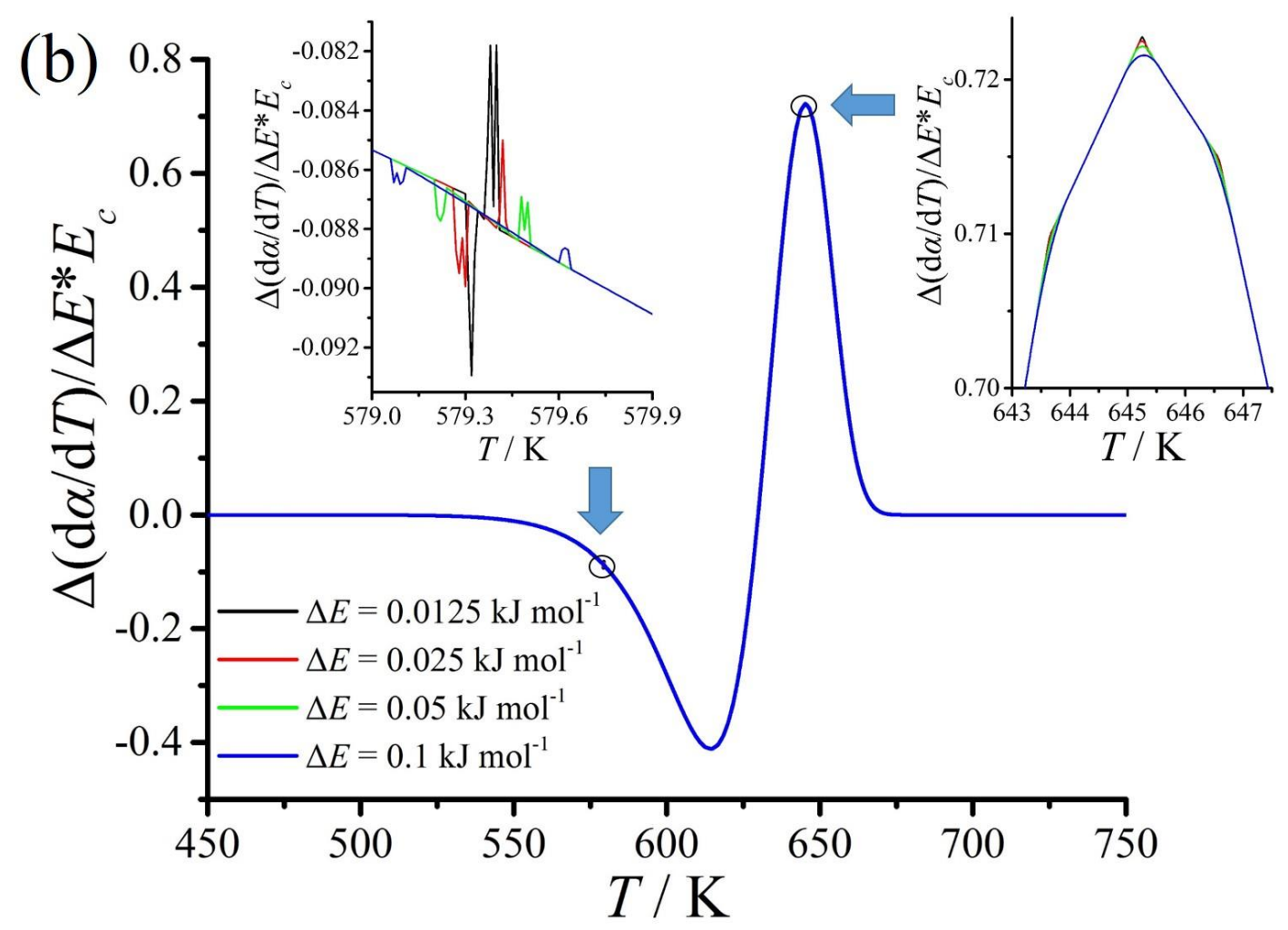

Figure 4. The effect of various $\Delta E$ values on the sensitivity of $\mathrm{d} \alpha / \mathrm{d} T$ with respect to $E$ at $E_{c}=$ $198.91 \mathrm{~kJ} \mathrm{~mol}^{-1}$ for the first order kinetic model
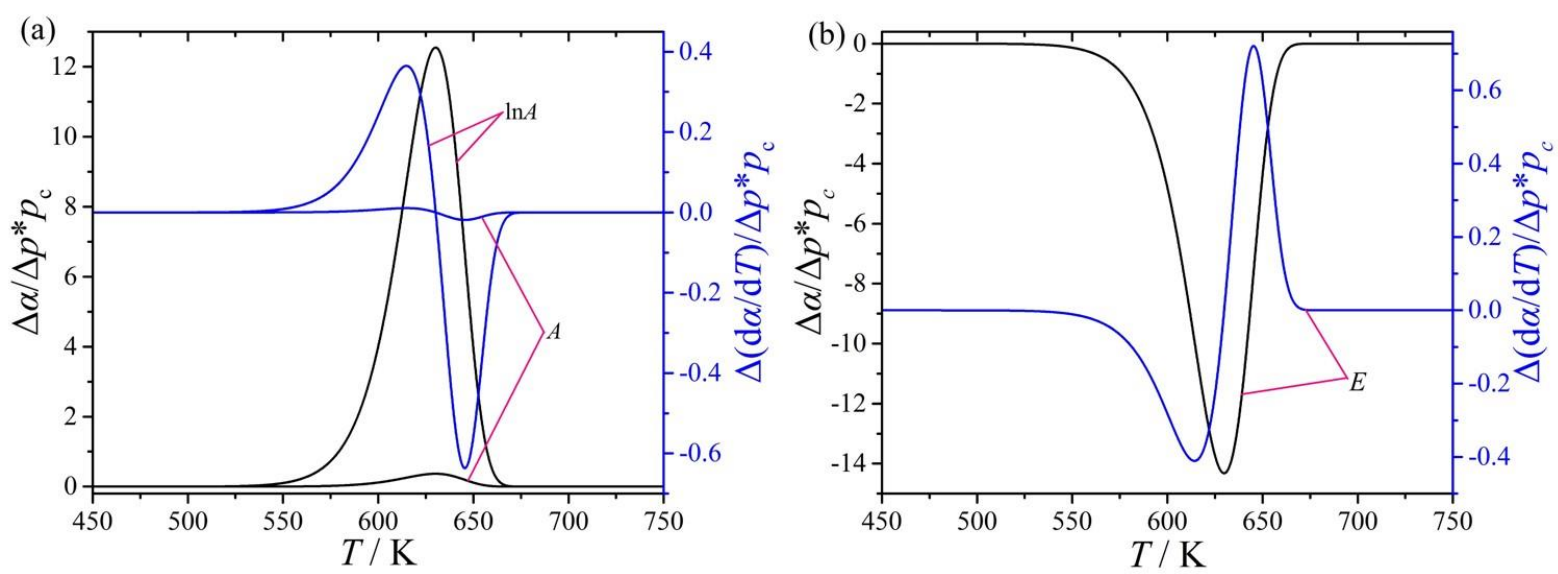

Figure 5. The relative sensitivities of $\alpha$ and $\mathrm{d} \alpha / \mathrm{d} T$ with respect to (a) $A$ and $\ln A$, and (b) $E$ at $A_{c}=6.46 \times 10^{14} \mathrm{~s}^{-1}$ and $E_{c}=198.91 \mathrm{~kJ} \mathrm{~mol}^{-1}$ for the first order kinetic model 
Table 1. The peak values of relative sensitivities of $\alpha$ and $\mathrm{d} \alpha / \mathrm{d} T$ for the first order kinetic model

\begin{tabular}{ccccccc}
\hline \multirow{2}{*}{ parameter } & $\begin{array}{c}\text { the peak value of } \\
\text { relative sensitivity } \\
\text { of } \alpha\end{array}$ & $\begin{array}{c}\text { the 1st peak value of } \\
\text { relative sensitivity of } \\
\mathrm{d} \alpha / \mathrm{d} T\end{array}$ & $\begin{array}{c}\text { the 2nd peak value of } \\
\text { relative sensitivity of } \\
\mathrm{d} \alpha / \mathrm{d} T\end{array}$ \\
\cline { 2 - 7 } & $T$ & $\Delta \alpha / \Delta p^{*} p_{c}$ & $T$ & $\Delta(\mathrm{d} \alpha / \mathrm{d} T) / \Delta p^{*} p_{c}$ & $T$ & $\Delta(\mathrm{d} \alpha / \mathrm{d} T) / \Delta p^{*} p_{c}$ \\
\hline$A$ & 630.3 & 0.368 & 614.9 & 0.0107 & 645.6 & -0.0187 \\
$\ln A$ & 630.2 & 12.5 & 614.8 & 0.365 & 645.6 & -0.637 \\
$E$ & 629.5 & -14.3 & 614.3 & -0.411 & 645.4 & 0.721 \\
\hline
\end{tabular}

\section{The $n$th order kinetic model and its sensitivity analysis}

The following $n$th order kinetic model is usually used to extend the applicability of the first order kinetic model [14]:

$$
\frac{\mathrm{d} \alpha}{\mathrm{d} T}=\frac{A}{\beta} e^{-E / R T}(1-\alpha)^{n}
$$

where $n$ is the reaction order.

Equation (14) with the initial condition (5) can be numerically solved by the classical Runge-Kutta fourth order algorithm. Figure 2 (d) shows the effect of the reaction order on the numerical results of the $n$th order kinetic model. It can be observed that (1) the increase of $n$ causes the slope of $\alpha-T$ curve to get less, (2) the height of the $\mathrm{d} \alpha / \mathrm{d} T-T$ curve peak decreases with the decrease of $n,(3)$ the temperature at the $\mathrm{d} \alpha / \mathrm{d} T-T$ curve peak remains constant with the variation of $n$.

The sensitivities of $A, E$, and $\ln A$ can be obtained according to the above equations (8) (13). The sensitivity of $n$ is calculated by using the following equations:

$$
\begin{gathered}
\frac{\Delta \alpha}{\Delta n}=\frac{\alpha(T, n+\Delta n)-\alpha(T, n-\Delta n)}{2 \Delta n} \\
\frac{\Delta \frac{\mathrm{d} \alpha}{\mathrm{d} T}}{\Delta n}=\frac{\frac{\mathrm{d} \alpha}{\mathrm{d} T}(T, n+\Delta n)-\frac{\mathrm{d} \alpha}{\mathrm{d} T}(T, n-\Delta n)}{2 \Delta n}
\end{gathered}
$$

Based on the calculated results, the optimal values of $\Delta A, \Delta \ln A, \Delta E, \Delta n$ for the $n$th order kinetic model are estimated: $\Delta A=10^{12} \mathrm{~s}^{-1}, \Delta \ln A=0.02, \Delta E=0.2 \mathrm{~kJ} \mathrm{~mol}^{-1}$, and $\Delta n=0.04$. The 
sensitivity analysis results of the $n$th order kinetic model with the optimal parameter changes are shown in Figure 6. The corresponding peak values of the temperature evolution of the relative sensitivities for the $n$th order kinetic model are listed in Table 2. From Figure 6 and Table 2, it can be obtained that the relative sensitivities of $\alpha$ and $\mathrm{d} \alpha / \mathrm{d} T$ with respect to $n$ and $A$ is slight, but the relative sensitivities of $\alpha$ and $\mathrm{d} \alpha / \mathrm{d} T$ with respect to $E$ and $\ln A$ is strong.
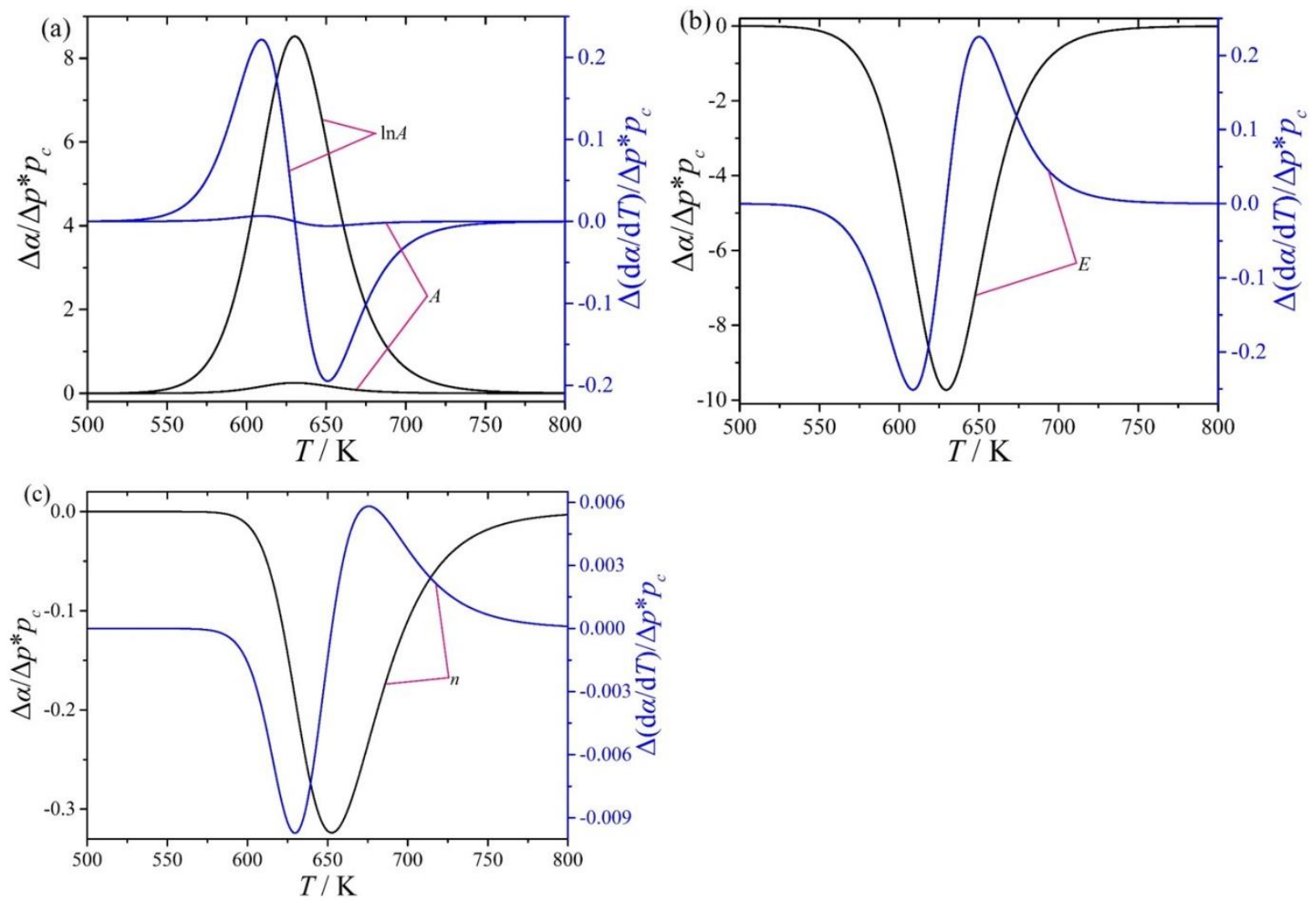

Figure 6. The relative sensitivities of $\alpha$ and $\mathrm{d} \alpha / \mathrm{d} T$ with respect to (a) $A$ and $\ln A$, (b) $E$, and (c) $n$ at $A_{c}=6.46 \times 10^{14} \mathrm{~s}^{-1}, E_{c}=198.91 \mathrm{~kJ} \mathrm{~mol}^{-1}$ and $n_{c}=2.0$ for the $n$th order kinetic model 
Table 2. The peak values of relative sensitivities of $\alpha$ and $\mathrm{d} \alpha / \mathrm{d} T$ for the $n$th order kinetic model

\begin{tabular}{ccccccc}
\hline \multirow{2}{*}{ parameter } & $\begin{array}{c}\text { the peak value of } \\
\text { relative sensitivity of }\end{array}$ & $\begin{array}{c}\text { the 1st peak value of } \\
\text { relative sensitivity of } \\
\mathrm{d} \alpha / \mathrm{d} T\end{array}$ & $\begin{array}{c}\text { the 2nd peak value of } \\
\text { relative sensitivity of } \\
\end{array}$ & \multicolumn{2}{c}{$\begin{array}{c}\alpha \\
\mathrm{n}\end{array}$} & \multicolumn{2}{c}{$\Delta / \Delta p^{*} p_{c}$} & $T$ & $\Delta(\mathrm{d} \alpha / \mathrm{d} T) / \Delta p^{*} p_{c}$ & $T$ & $\Delta(\mathrm{d} \alpha / \mathrm{d} T) / \Delta p^{*} p_{c}$ \\
\hline$A$ & 630.3 & 0.250 & 609.4 & 0.00650 & 650.9 & -0.00572 \\
$\ln A$ & 630.2 & 8.53 & 609.3 & 0.222 & 650.8 & -0.195 \\
$E$ & 629.5 & -9.73 & 608.6 & -0.251 & 650.0 & 0.225 \\
$n$ & 652.4 & -0.324 & 629.4 & -0.00973 & 674.8 & 0.00581 \\
\hline
\end{tabular}

\section{Conclusions}

(1) The local sensitivity analysis of the first and $n$th order kinetic model for cellulose pyrolysis was performed based on the finite differential method. The optimal values of parametric changes were obtained: $\Delta E=0.2 \mathrm{~kJ} \mathrm{~mol}^{-1}, \Delta A=10^{12} \mathrm{~s}^{-1}, \Delta \ln A=0.02$ and $\Delta n=0.04$.

(2) For the first order kinetic model, the activation energy and the natural logarithm of the frequency factor affected the conversion and conversion rate more strongly.

(3) The $n$th order kinetic model was slightly sensitive to the reaction order, while strongly sensitive to the activation energy and the logarithm of the frequency factor.

(4) Compared with the frequency factor, the natural logarithm of the frequency factor was a better choice in the parameter estimation.

(5) Such sensitivity analysis occurring in cellulose pyrolysis provides qualitative and quantitative insights for biomass pyrolysis kinetics.

\section{Acknowledgement}

Financial support from Participation in Research Program at Shanghai Jiao Tong University (Project NO. T150PRP31027) is greatly acknowledged. 


\section{References}

[1] Sindhu R, Binod P, Pandey A. Biological pretreatment of lignocellulosic biomass - An overview. Bioresource Technology 2016;199:76.

[2] Hassan H, Lim JK, Hameed B. Recent progress on biomass co-pyrolysis conversion into high-quality bio-oil Bioresource Technology 2016;221:645.

[3] Muley PD, Henkel C, Abdollahi KK, Marculescu C, Boldor D. A critical comparison of pyrolysis of cellulose, lignin, and pine sawdust using an induction heating reactor. Energy Conversion and Management 2016;117:273.

[4] Sánchez-Jiménez PE, Pérez-Maqueda LA, Perejón A, Pascual-Cosp J, Benítez-Guerrero M, Criado JM. An improved model for the kinetic description of the thermal degradation of cellulose. Cellulose 2011;18:1487.

[5] Lédé J. Cellulose pyrolysis kinetics: An historical review on the existence and role of intermediate active cellulose. Journal of Analytical and Applied Pyrolysis 2012;94:17.

[6] Paulik J, Paulik F. Complex thermoanalytical method for the simultaneous recording of T, TG, DTG, DTA, TGT, DTGT, TD and DTD curves Part I. Development and characterization of equipment. Thermochimica Acta 1971;3:13.

[7] Paulik F, Bessenyey-Paulik E, Walther-Paulik K. Transformation-governed heating technique in thermal analysis. Part III. Normalisation of experimental conditions. Journal of Thermal Analysis and Calorimetry 2002;69:339.

[8] Paulik F, Bessenyey-Paulik E, Walther-Paulik K. Differential thermal analysis under quasiisothermal, quasi-isobaric conditions (Q-DTA) Part II. Water evaporation and the decomposition mechanism of compounds with structural and crystal water. Thermochimica Acta 2004;424:75.

[9] White JE, Catallo WJ, Legendre BL. Biomass pyrolysis kinetics: A comparative critical review with relevant agricultural residue case studies. Journal of Analytical and Applied Pyrolysis 2011;91:1.

[10] Várhegyi G. Aims and methods in non-isothermal reaction kinetics. Journal of Analytical and Applied Pyrolysis 2007;79:278.

[11] Saltelli A, Tarantola S, Campolongo F, Ratto M. Sensitivity Analysis in Practice: A Guide to Assessing Scientific Models: Wiley; 2004.

[12] Saltelli A, Tarantola S, Campolongo F. Sensitivity Analysis as an Ingredient of Modeling. Statistical Science 2000;15:377. 
[13] Mezaki R, Kittrell JR. Parametetric sensitivity in fitting nonlinear kinetic models. Industrial \& Engineering Chemistry 1967;59:63.

[14]Burnham AK, Zhou X, Broadbelt LJ. Critical review of the global chemical kinetics of cellulose thermal decomposition. Energy \& Fuels 2015;29:2906.

[15] Antal MJ, Varhegyi G, Jakab E. Cellulose pyrolysis kinetics: revisited. Industrial \& Engineering Chemistry Research 1998;37:1267.

[16] Lin Y-C, Cho J, Tompsett GA, Westmoreland PR, Huber GW. Kinetics and mechanism of cellulose pyrolysis. The Journal of Physical Chemistry C 2009;113:20097.

[17] Bradbury AGW. A kinetic model for pyrolysis of cellulose. Journal of applied polymer science 1979;23:3271.

[18] Nguyen-Tuan L, Lahmer T, Datcheva M, Schanz T. Global and local sensitivity analyses for coupled thermo-hydro-mechanical problems. International Journal for Numerical and Analytical Methods in Geomechanics 2016:doi: 10.1002/nag.2573.

[19] Santos K, Lobato F, Lira T, Murata V, Barrozo MA. Sensitivity analysis applied to independent parallel reaction model for pyrolysis of bagasse. Chemical Engineering Research and Design 2012;90:1989.

[20] Xavier TP, Lira TS, Schettino Jr. MA, Barrozo MAS. A study of pyrolysis of macadamia nut shell: parameteric sensitivity analysis the IPR model. Brazilian Journal of Chemical Engineering 2016;33:115.

[21]Cai J. A distributed activation energy model for the pyrolysis of lignocellulosic biomass. Green chemistry 2013;15:1331.

[22] Carrier M, Auret L, Bridgwater A, Knoetze JH. Using apparent activation energy as a reactivity criterion for biomass pyrolysis. Energy \& Fuels 2016;30:7834.

[23] Sørensen OT, Rouquerol J. Sample Controlled Thermal Analysis: Origin, Goals, Multiple Forms, Applications and Future: Springer US; 2013.

[24] Cai J, Yao F, Yi W, He F. New temperature integral approximation for nonisothermal kinetics. AIChE Journal 2006;52:1554.

[25] JOHANSEN S. The Welch-James approximation to the distribution of the residual sum of squares in a weighted linear regression. Biometrika 1980;67:85.

[26] Morgan J, Tatar J. Calculation of the residual sum of squares for all possible regressions. Technometrics 1972;14:317.

[27] Saltelli A, Ratto M, Tarantola S, Campolongo F. Sensitivity analysis for chemical models. Chemical Reviews 2005;105:2811. 
[28] Saltelli A, Chan K, Scott EM. Sensitivity Analysis: Wiley; 2009. 